NON-MAGNETIC CATACLYSMIC VARIABLES 


\title{
THE NOVA-LIKE VARIABLES
}

\author{
VIK DHILLON \\ Royal Greenwich Observatory, La Palma
}

\begin{abstract}
We review optical observations and theoretical models of the non-magnetic nova-like variables (UX UMa, VY Scl and SW Sex stars). A brief discussion of the classification scheme is followed by a statistical overview of the observed properties. The most important features of each of the sub-classes are then reviewed, concluding with a summary of the theoretical models invoked to understand these systems.
\end{abstract}

\section{Classification}

Nova-like variables are defined as cataclysmic variable stars (CVs) which have never been observed to undergo nova or dwarf nova-type outbursts. Such a crude definition encompasses a wide variety of objects which can be divided into two distinct groups; those which are believed to accrete via magnetic field lines - AM Her stars (or polars), DQ Her stars and intermediate polars - and those which are believed to accrete via an accretion disc - UX UMa stars, VY Scl stars (or anti-dwarf novae), SW Sex stars and AM CVn stars (or double-degenerates). The magnetic nova-likes have been reviewed elsewhere in this volume. The subject of this review will be the optical characteristics of non-magnetic nova-likes (NMNLs), with the exception of the AM CVn stars which are outside the scope of this paper.

There is little agreement on the classification scheme for NMNLs in the literature. Perhaps the most generally accepted scheme adheres to the following rules: if a nova-like shows no evidence for magnetic accretion and is not recognized to be a double-degenerate, it is classed as a UX UMa star. A UX UMa star which is observed to show states of low brightness becomes a VY Scl star. A number of UX UMa and VY Scl stars with high inclinations and periods of $3 \ldots 4 \mathrm{~h}$, show single-peaked emission lines which remain (largely) unobscured during primary eclipse and which exhibit transient absorption features and distorted radial velocity curves. These systems

A. Evans and J. H. Wood (eds.), Cataclysmic Variables and Related Objects, 3-12.

(c) 1996 Kluwer Academic Publishers. Printed in the Netherlands. 


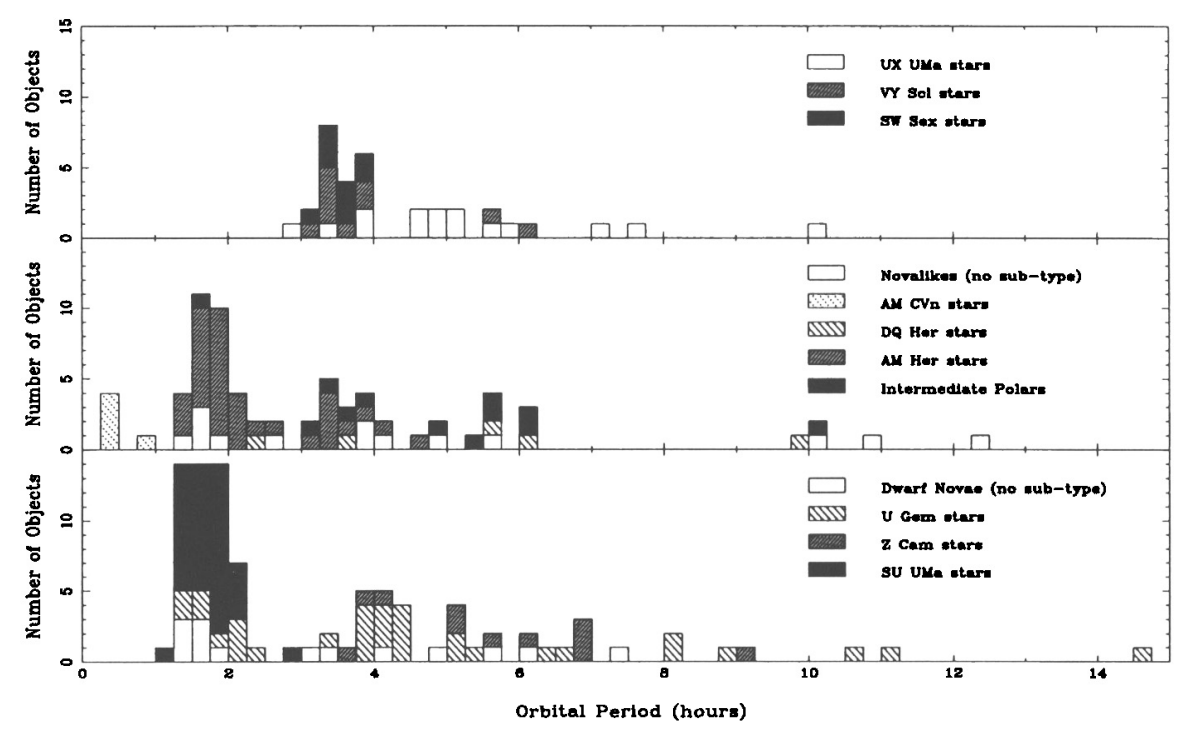

Figure 1. The orbital period distribution of CVs. Data from [31].

were classed as SW Sex stars by Thorstensen et al. [44]. This classification is controversial, however, since SW Sex stars can only be recognized by their spectroscopic properties, whereas other classes of CV are traditionally classified by their photometric variations. Amongst other things, this can lead to confusion, since a star can then be both a VY Scl star and an SW Sex star. In addition, it is possible that the SW Sex stars are simply the high inclination counterparts of other NMNLs, in which case a separate classification is not justified. Bearing these caveats in mind, it is nevertheless sometimes convenient to be able to refer to these objects as SW Sex stars and this term will be used in the present review.

\section{Statistical overview}

Of the $703 \mathrm{CVs}$ known, 104 are classed as nova-like variables [9]. Of these, 21 are UX UMa stars and 13 VY Scl stars. Arguably, up to 6 of these UX UMa stars and 3 of these VY Scl stars exhibit the SW Sex phenomenon [31]. When plotted in Galactic coordinates [23], it is immediately apparent that nova-likes are found in equal numbers in the Galactic plane and at high galactic latitudes, with no tendency for their numbers to increase towards the Galactic centre. The implication is that, like the dwarf novae, novalikes are nearby and, given their low apparent brightness, they must also be intrinsically faint. These conclusions are confirmed by determinations of distances (of order hundreds of parsecs) and absolute magnitudes $\left(M_{V} \sim 4\right)$ of nova-likes [47]. The actual Galactic distributions and space densities are, however, much more contentious subjects: see la Dous [23]. 


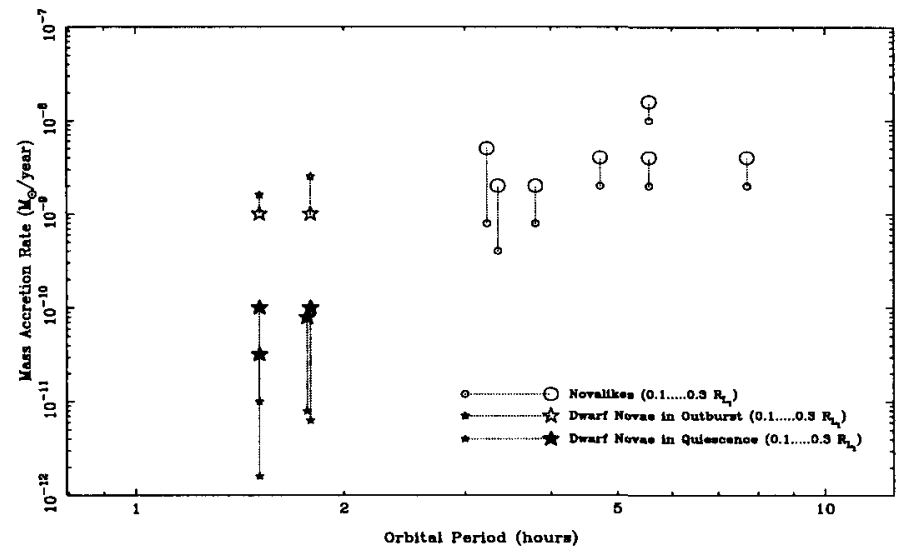

Figure 2. The dependence of mass accretion rate at disc radii $0.1 R_{L_{1}}$ and $0.3 R_{L_{1}}$ on orbital period for dwarf novae (in outburst and quiescence) and NMNLs.

Fig. 1 shows the orbital period distribution of CVs. It can be seen that the NMNLs are tightly grouped in the $3 \ldots 4 \mathrm{~h}$ period range and there are no NMNLs below the $2 \ldots 3 \mathrm{~h}$ period gap. The dwarf novae, on the other hand, are found both above and below the period gap and there appears to be a dearth of dwarf novae in the $3 \ldots 4 \mathrm{~h}$ period range favoured by the NMNLs. The implications of this result will be discussed in Sect. 4 .

One of the most important distinctions between NMNLs and other CVs can be found by comparing their mass accretion rates. Following Horne [22], Fig. 2 plots the mass accretion rate derived from eclipse mapping experiments as a function of binary period for a number of NMNLs and dwarf novae in outburst and quiescence. It is evident that both NMNLs and dwarf novae in outburst accrete mass at similar rates and at a much higher rate than dwarf novae in quiescence. The accretion rate also appears to increase slightly with orbital period.

A discrepancy between steady-state accretion (in which the mass accretion rate is constant throughout the disc) and the observed accretion rates can also be inferred from Fig. 2. The mass accretion rate appears to decrease slightly with radius in the dwarf novae discs during outburst. This is expected since material should be draining from the disc as it declines from outburst. The NMNLs, however, all show mass accretion rates that increase with radius in the disc. The increase is low for the longer period NMNLs, but becomes significant for systems in the $3 \ldots 4 \mathrm{~h}$ period range. The latter result has been inferred from the eclipse maps of Rutten, van Paradijs \& Tinbergen [36], which show that the radial temperature dependences in the inner disc regions of SW Sex stars are much flatter than the $T \propto R^{-3 / 4}$ relation predicted by steady-state accretion theory. The implication is that the central regions of these discs may be releasing some of the accretion energy in a non-radiative form, and it seems likely that this result is linked 
to the SW Sex phenomenon which requires an extra component of light that remains visible during primary eclipse (see Sect. 4).

\section{Observed properties}

The long-term light curves of NMNLs are as varied and complex as those exhibited by novae and dwarf novae. For example, some VY Scl stars (e.g. V794 Aql [19]) show deep low states of $\sim 3 \mathrm{mag}$, preceded by a number of shallower low states with saw-toothed light curves. Other VY Scl stars (e.g. DW UMa - also an SW Sex star [18]), show even deeper low states of much longer duration (typically years). Inevitably, there are also VY Scl stars which show both types of behaviour (e.g. MV Lyr [34]). As well as low states, however, some NMNLs also show outbursts; Honeycutt et al. [20] observed a 1 mag outburst in the VY Scl star KR Aur, while Still, Dhillon \& Jones [42], observed the UX UMa star RW Tri during a $3.5 \mathrm{mag}$ outburst. It is not known how often such eruptions occur in NMNLs, and whether or not they are related to nova or dwarf nova-type outbursts is unclear.

Other types of long-term photometric variations have also been observed in NMNLs. For example, RW Tri was observed by Honeycutt et al. [20] to show sinusoidal-like brightness variations of $0.5 \mathrm{mag}$ with a period of $25 \mathrm{~d}$. RW Tri and UX UMa also show aperiodic variations in their orbital period $[35],[42]$. There have been suggestions that these phenomena might be the result of magnetic cycles within the secondary star: see [1] and references therein.

Moving to variations on orbital time-scales, the light curves of high inclination NMNLs exhibit smooth, round-bottomed eclipses, often with a pronounced egress shoulder and orbital hump due to the bright spot (see Fig. 5). Similar orbital light curves are exhibited by dwarf novae in outburst. The eclipse light curves of NMNLs can be variable from cycle to cycle, both in depth and in shape (e.g. PX And [44]). Eclipse maps show that the discs are generally symmetric, much brighter in their centres than their edges, and have bright spots whose strengths vary considerably from object to object [36].

On time-scales of minutes, all NMNLs show stochastic flickering with amplitudes of up to several tenths of a magnitude. By analysing the behaviour of the flickering in RW Tri during eclipse, Horne \& Stiening [21] showed that the entire disc participates in the flickering; it is not known whether this flickering is the result of irradiation from near the white dwarf, or energy released at localized sites in the disc. On even shorter time-scales, quasi-periodic oscillations (QPOs) with periods of tens of seconds are sometimes seen in the light curves of NMNLs (and dwarf novae during outburst), 

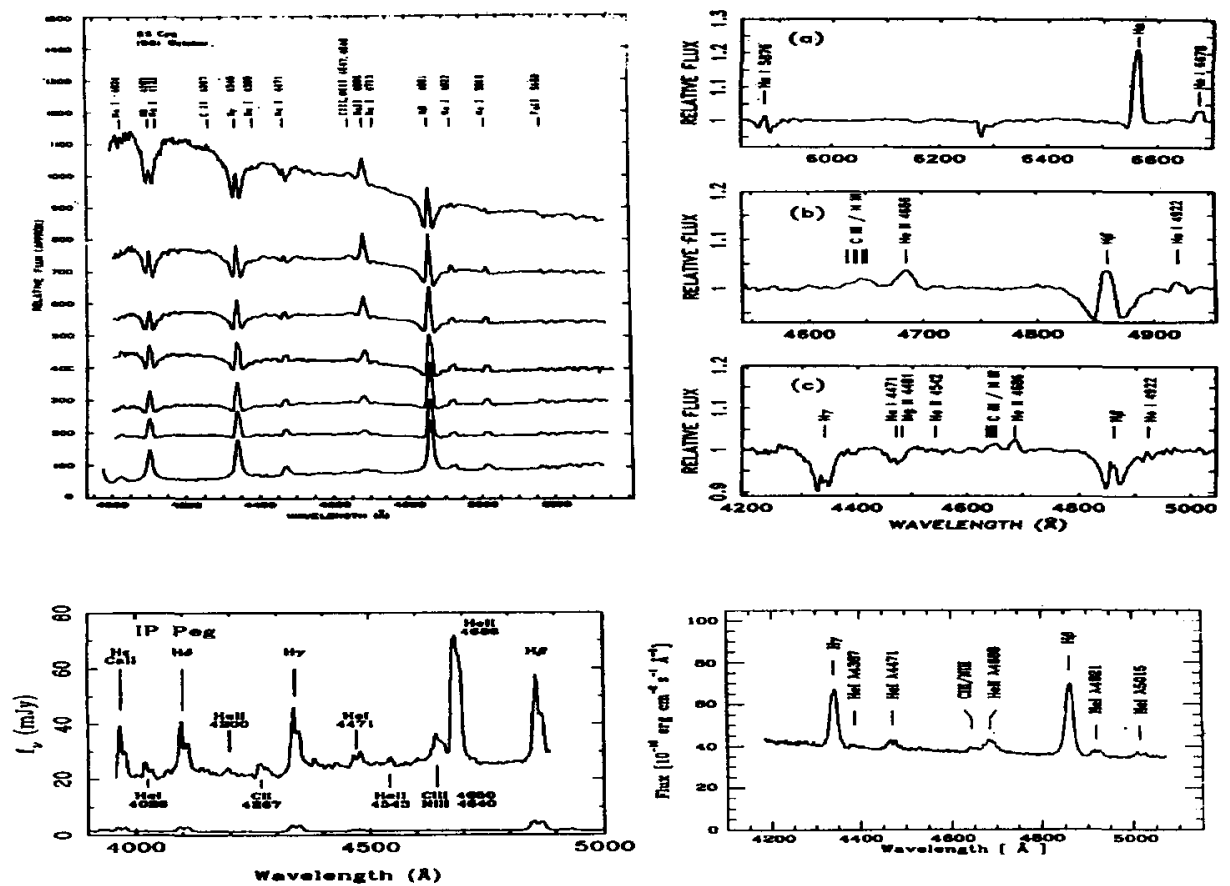

Figure 3. A comparison of the spectra of dwarf novae and NMNLs. Clockwise from top left: the low inclination dwarf nova SS Cyg in outburst and quiescence [15]; the low inclination UX UMa star IX Vel [2]; the high inclination SW Sex star WX Ari [3]; the high inclination dwarf nova IP Peg in outburst and quiescence [27].

e.g. V3885 Sgr [46] and UX UMa [28]. Petterson [30] successfully modelled the systematic changes observed in the QPO phases during eclipse with an accretion disc reflecting radiation from a rotating source on or near the surface of the white dwarf. Although various models for the source of radiation have been invoked (e.g. bright spots on the white dwarf; non-radial pulsations; bright spots at the inner edge of the accretion disc), their true nature remains uncertain.

NMNLs exhibit the same variety in their optical spectra as do dwarf novae in outburst and quiescence, ranging from pure emission line to almost pure absorption line spectra. In Fig. 3, representative spectra of high and low inclination dwarf novae in outburst and quiescence are displayed alongside spectra of high and low inclination NMNLs. The similarities are striking. Low inclination dwarf novae in outburst show strong absorption lines, as do low inclination NMNLs. High inclination dwarf novae in outburst show strong emission lines, as do high inclination NMNLs. This strong correlation between orbital inclination and the strength of the emission lines has been demonstrated for classical novae by Warner [47] and has been noted for dwarf novae in outburst by Robinson, Marsh \& Smak [33]. 


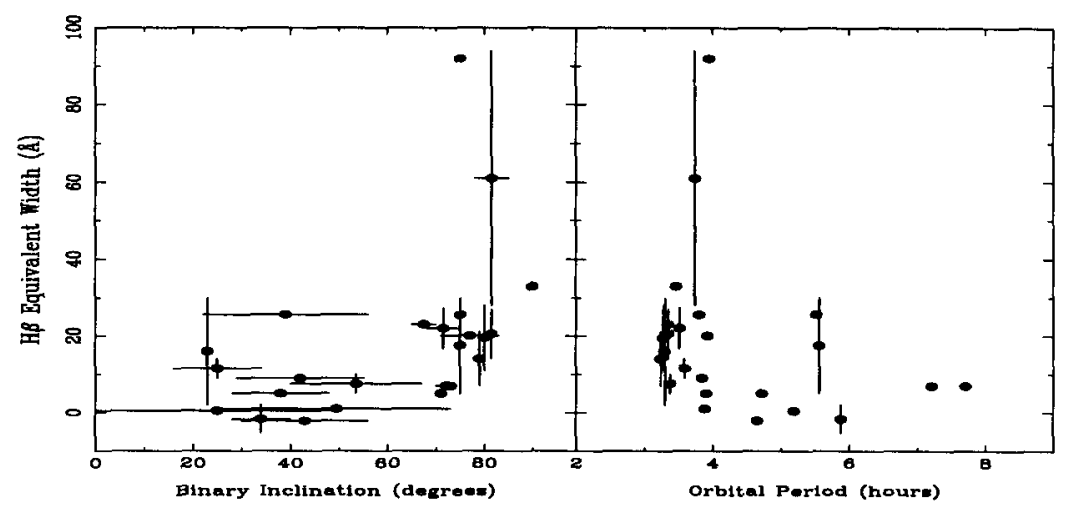

Figure 4. Binary inclination and orbital period versus the equivalent width of $\mathrm{H} \beta$ in a sample of $24 \mathrm{UX}$ UMa, VY Scl and SW Sex-type nova-like variables.

Fig. 4, which plots the equivalent width of $\mathrm{H} \beta$ versus the orbital inclination, shows that the same correlation exists in NMNLs; low inclination NMNLs generally show absorption lines (often with strong emission cores, which is why the equivalent widths are rarely negative), whereas high inclination NMNLs show emission lines. The reason that this correlation exists is due to dilution: at high inclinations the flux in the optically-thick continuum of the accretion disc is reduced by projection and limb darkening, so there is less dilution of the emission line flux. Fig. 4 also shows that the equivalent width of $\mathrm{H} \beta$ increases with decreasing orbital period in NMNLs. Hessman [14] and Echevarria [11] found a similar correlation exists for all CVs. This effect can be understood in terms of the correlation between line strength and absolute magnitude found by Patterson [29]: as the accretion disc becomes brighter the emission lines are diluted by the optically thick continuum flux from the disc and hence their equivalent widths decrease. Since CVs with longer orbital periods tend to have higher rates of mass transfer (see Fig. 2), their emission line strengths decrease.

The remainder of this section will review the spectroscopic properties specific to the UX UMa, SW Sex and VY Scl sub-classes. Perhaps the best understood class of NMNLs are the UX UMa stars. That this is the case has been verified in some detail by the spectral eclipse-mapping results of Rutten et al. [37]. They reconstructed the spectra of different parts of the accretion disc in UX UMa and found an inner disc and bright spot with a blue continuum and Balmer absorption lines and an outer disc with a red continuum and Balmer emission lines. This is qualitatively what is expected from accretion discs which are hot and optically thick in their centres, and cool and optically thin in their outer regions [48]. In addition, theoretical spectra now appear to be giving encouraging fits to the observed continua of UX UMa stars [40].

The same agreement with theory cannot be said to exist for SW Sex 

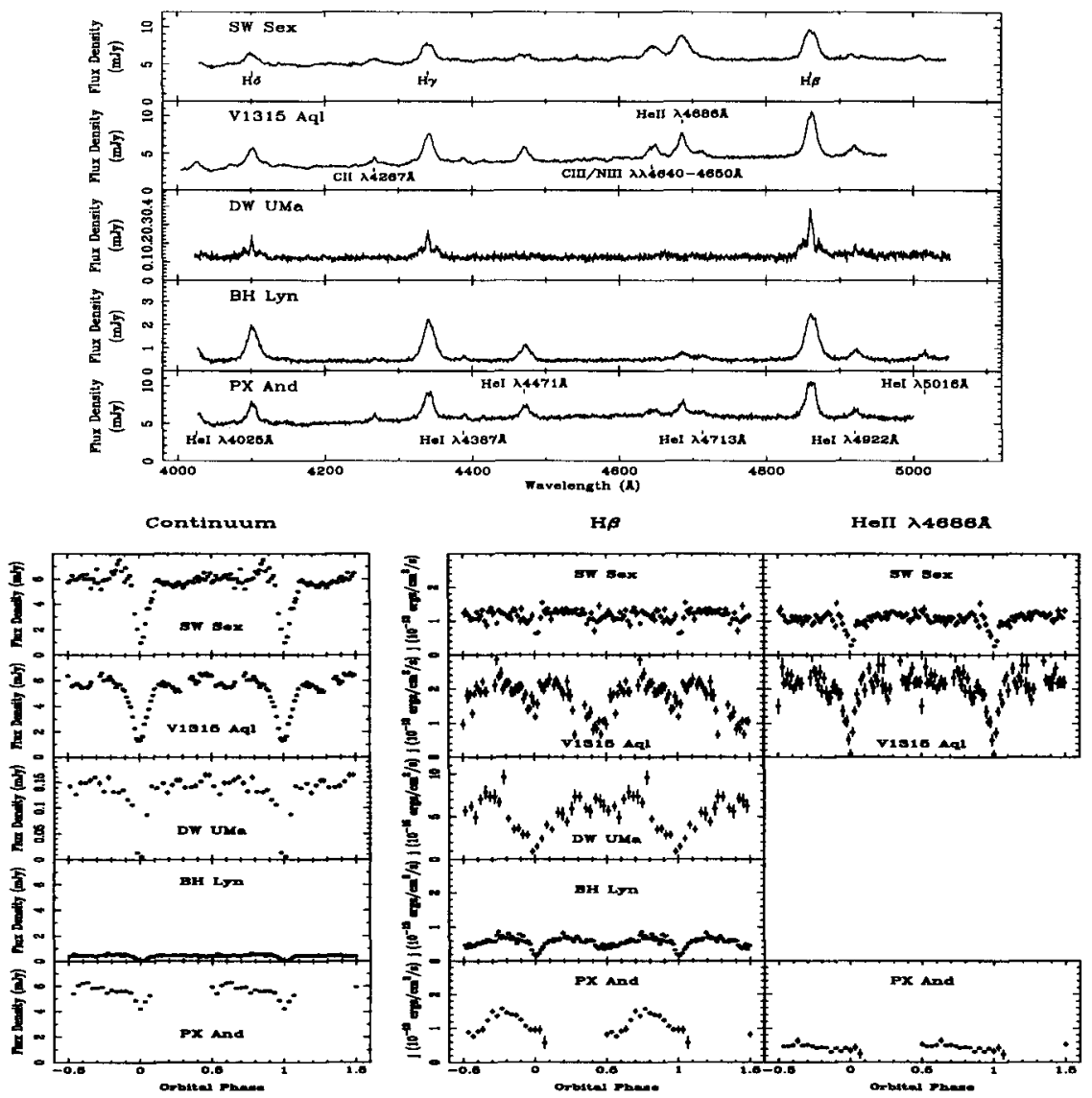

Figure 5. Spectra and light curves of 5 SW Sex stars. From top to bottom: SW Sex [4], V1315 Aql [5], DW UMa (low state [7]), BH Lyn (low state [6]) and PX And [43].

stars. This group of stars are all high inclination $\mathrm{NMNLs}^{1}$ with periods in the range $3 . .4 \mathrm{~h}$. The spectra of $5 \mathrm{SW}$ Sex stars and their continuum and emission line light curves are displayed in Fig. 5. Note that the spectra and light curves of DW UMa and BH Lyn were obtained during low states (see below). All SW Sex stars exhibit strong, single-peaked Balmer, He I and He II emission lines which, with the exception of He II, remain largely unobscured during primary eclipse. This is in stark contrast to standard accretion disc theory which predicts that emission lines from high inclination discs should appear double peaked and be eclipsed once every orbital period. In addition, the emission lines in SW Sex stars show strong absorption features around phase 0.5 and their radial velocity curves exhibit significant phase shifts relative to photometric conjunction. As shall be discussed in

\footnotetext{
${ }^{1}$ Even WX Ari, previously thought to be low inclination, shows grazing eclipses [13]
} 
Sect. 4 , these observational constraints have yet to be fully satisfied by a single theoretical model.

The VY Scl stars in their normal state are indistinguishable from UX UMa or SW Sex stars. In their low states, however, which are due to a decrease in the mass accretion rate, VY Scl stars completely change in appearance. An example of typical VY Scl-star behaviour has been presented by Dhillon, Jones \& Marsh [7], who observed DW UMa in a $\sim 3 \mathrm{mag}$ low state; the resulting spectrum and light curves are shown in Fig. 5. It can be seen that, in contrast to the normal state, there are no high-excitation emission lines present. The Balmer lines are dominated by strong, narrow emission spikes superposed upon faint wings. Dhillon et al. [7] showed that the emission spikes originate on the secondary star and the line wings show evidence for an accretion disc origin. By measuring the continuum eclipse width, they also found that the accretion disc was smaller than during the normal state. Using this same data in conjunction with Roche tomography, Rutten \& Dhillon [38] were able to map the Balmer line intensity distribution on the surface of the secondary in DW UMa and showed strongly enhanced emissivity on the inner face of the secondary star. A similar result was also obtained by Still et al. [42] in their study of RW Tri during a $\sim 3.5$ mag high state, suggesting that irradiation by the accretion disc plays an important role in NMNLs.

\section{Theoretical models}

The observed similarities between NMNLs and dwarf novae in outburst suggest that NMNLs might be successfully modelled by canonical CVs with steady-state accretion discs in which the mass transfer rate is sufficiently high to prevent disc instability-type outbursts. But can this model explain all of the observed NMNL phenomena, and in particular, the low states, the orbital period distribution, the departures from steady-state accretion in the inner disc regions and the SW Sex phenomenon? In this final section we will show that a number of modifications to the canonical model are required in order to approach an understanding of the NMNLs, a conclusion which has also been reached by la Dous [24].

Turning first to the SW Sex phenomenon, it is clear that a successful model will have to include some component of line emission from above the orbital plane in order to explain the lack of eclipse in the emission lines. For this reason, the Stark broadening model of Lin, Williams \& Stover [25] and the bright-spot overflow model of Hellier \& Robinson [12] are unlikely to be correct. However, the latter model is very successful in explaining many of the other properties of SW Sex stars, such as the phase 0.5 absorption. This suggests that a model with a bright-spot overflow operating in con- 
junction with some form of accretion disc wind ([8]; [16]; [17]), magnetic accretion column [49], or magnetically driven outflow ([45]; [50]) might be able to explain all of the observed phenomena. The requirement that some mechanism drives material out of the orbital plane is also consistent with the observed departures from steady-state accretion in the inner discs of these systems and provides an explanation for the redder UV spectra of NMNLs compared to dwarf novae in outburst [45].

The first attempt to explain the observation of low states in VY Scl stars was made by Robinson et al. [32]. They noted the period grouping of VY Scl stars and speculated that the low states are a consequence of their imminent entry into the period gap. In this model, the magnetic braking which drives mass transfer ceases as a result of the secondary star becoming fully convective at periods immediately above the gap [41]. However, Livio \& Pringle [26] showed that this model cannot account for the low states in VY Scl stars due to the disparate time-scales - it takes VY Scl stars of order $10 \ldots 100 \mathrm{~d}$ to enter a low state whereas it would take $10000 \mathrm{yr}$ or more to respond to a sudden cessation in the mechanism driving the mass transfer. They proposed an alternative model where star spots covering the $\mathrm{L}_{1}$ point block the mass transfer and hence cause low states. It may be possible to test this model using Roche tomography [38].

Using the eruptive characteristics of CVs to infer relative mass transfer rates, Shafter [39] concluded that magnetic braking models have severe difficulties accounting for the orbital period distribution of CVs, in particular the observed dearth of dwarf novae with orbital periods immediately above the period gap and the dominance of NMNLs in this same period range. Intriguingly, the star spot model of Livio \& Pringle [26] can also be used to explain this orbital period distribution. The dwarf novae, with lower mass transfer rates, are more easily interrupted by the star spot mechanism because the density and pressure are correspondingly lower at the $\mathrm{L}_{1}$ point. As the periods decrease, the magnetic fields and their covering factors might be expected to increase, so that if mass transfer occurs it can only do so at a relatively high. rate. In their picture, therefore, the low mean mass transfer rate systems (dwarf novae) become detectable as high mass transfer rate systems (NMNLs) as they approach the period gap.

But why do NMNLs have higher mean mass transfer rates than dwarf novae? This is ultimately the most important question of all, the answer to which remains uncertain. According to the hibernation model for the cyclical evolution of CVs (see [10] and references therein), it is due to the fact that NMNLs are post-novae whose outbursts we have missed. Only time will tell if this theory is correct.

\section{References}

1. Applegate, J.H., 1992, Ap. J., 385, 621 
2. Beuermann, K., Thomas, H.-C., 1990, A\&A, 230, 326

3. Beuermann, K., Thorstensen, J.R., Schwope, A.D., et al., 1992, A\&A, 256, 442

4. Dhillon, V.S., 1990, D.Phil. thesis, University of Sussex

5. Dhillon, V.S., Marsh, T.R., Jones, D.H.P., 1991, MNRAS, 252, 342

6. Dhillon, V.S., Jones, D.H.P., Marsh, T.R., Smith, R.C., 1992, MNRAS, 258, 225

7. Dhillon, V.S., Jones, D.H.P., Marsh, T.R., 1994, MNRAS, 266, 859

8. Dhillon, V.S., Rutten, R.G.M., 1995, MNRAS, 277, 777

9. Downes, R.A., Shara, M.M., 1993, PASP, 105, 127

10. Duerbeck, H.W., 1993, in "Cataclysmic Variables and Related Physics", eds O. Regev, G. Shaviv, Ann. Israel Phys. Soc., 10, 77

11. Echevarria, J., 1988, MNRAS, 233, 513

12. Hellier, C., Robinson, E.L., 1994, Ap. J., 431, L107

13. Hellier, C., Ringwald, F.A., Robinson, E.L., 1995, A\&A, 289, 148

14. Hessman, F.V., 1985, Ph.D. thesis, University of Texas at Austin

15. Hessman, F.V., Robinson, E.L., Nather, R.E., Zhang, E., 1984, Ap. J., 286, 747

16. Hoare, M.G., 1994, MNRAS, 267, 153

17. Honeycutt, R.K., Schlegel, E.M., Kaitchuck, R.H., 1986, Ap. J., 302, 388

18. Honeycutt, R.K., Livio, M., Robertson, J.W., 1993, PASP, 105, 922

19. Honeycutt, R.K., Cannizzo, J.K., Robertson, J.W., 1994, Ap. J., 425, 835

20. Honeycutt, R.K., Robertson, J.W., Turner, G.W., et al., 1994, in "Interacting Binary Stars", ed. A. Shafter, ASP Conf. Ser. 56, 277

21. Horne, K., Stiening, R.F., 1985, MNRAS, 216, 933

22. Horne, K., 1993, in "Acc. Disks in Comp. Stell. Sys.", ed. J.C. Wheeler, p117

23. la Dous, C., 1993a, in 'CVs and Related Objects', NASA Monograph, p14

24. la Dous, C., 1993b, in "Cataclysmic Variables and Related Physics", eds O. Regev, G. Shaviv, Ann. Israel Phys. Soc., 10, 39

25. Lin, D.N.C, Williams, R.E., Stover, R.J., 1988, Ap. J., 327, 234

26. Livio, M., Pringle, J.E., 1994, Ap. J., 427, 956

27. Marsh, T.R., Horne, K., 1990, Ap. J., 349, 593

28. Nather, R.E., Robinson, E.L., 1974, Ap. J., 190, 637

29. Patterson, J., 1984, Ap. J. Supp., 54, 443

30. Petterson, J.A., 1980, Ap. J., 241, 247

31. Ritter, H., Kolb, U., 1995, in "X-ray Binaries", eds. Lewin, W.H.G., et al., CUP, Cambridge, p578

32. Robinson, E.L., Barker, E.S., Cochran, A.L., et al., 1981, Ap. J., 251, 611

33. Robinson, E.L., Marsh, T.R., Smak. J.I., 1993, in "Acc. Disks in Comp. Stell. Sys.", ed. J.C. Wheeler, p75

34. Rosino, L., Romano, G., Marziani, P., 1993, PASP, 105, 51

35. Rubenstein, E.P., Patterson, J., Africano, J.L., 1991, PASP, 103, 1258

36. Rutten, R.G.M., van Paradijs, J., Tinbergen, J., 1992, A\&A, 260, 213

37. Rutten, R.G.M., Dhillon, V.S., Horne, K., Kuulkers, E., 1994, A\&A, 283, 441

38. Rutten, R.G.M., Dhillon, V.S., 1994, A\&A, 288, 773

39. Shafter, A.W., 1992, Ap. J., 394, 268

40. Shaviv, G., Wehrse, R., 1993, in "Acc. Disks in Comp. Stell. Sys.”, ed. J.C. Wheeler, p148

41. Spruit, H.C., Ritter, H., 1983, A\&A, 124, 267

42. Still, M.D., Dhillon, V.S., Jones, D.H.P., 1995, MNRAS, 273, 849

43. Still, M.D., Dhillon, V.S., Jones, D.H.P., 1995, MNRAS, 273, 863

44. Thorstensen, J.R., Ringwald, F.A., Wade, R.A., et al., 1991, AJ, 102, 272

45. Tout, C.A., Pringle, J.E., la Dous, C., 1993, MNRAS, 265, 5p

46. Warner, B., 1973, MNRAS, 162, 189

47. Warner, B., 1987, MNRAS, 227, 23

48. Williams, R.E., 1980, Ap. J., 235, 939

49. Williams, R.E., 1989, AJ, 97, 1752

50. Wynn, G.A., King, A.R., 1996, these proceedings, p161 